\title{
Dopaminergic drug treatment remediates exaggerated cingulate prediction error responses in obsessive-compulsive disorder
}

\author{
Graham K. Murray ${ }^{1,2,3} \cdot$ Franziska Knolle $^{1,2}$ (D) $\cdot$ Karen D. Ersche ${ }^{1,2} \cdot$ Kevin J. Craig $^{1,2} \cdot$ Sanja Abbott ${ }^{2,4,5}$. \\ Shaila S. Shabbir ${ }^{6}$. Naomi A. Fineberg ${ }^{7}$. John Suckling ${ }^{1,2} \cdot$ Barbara J. Sahakian $^{1,2}$ • Edward T. Bullmore ${ }^{1,2,3}$. \\ Trevor W. Robbins ${ }^{2,4}$
}

Received: 23 January 2019 / Accepted: 30 May 2019 / Published online: 14 June 2019

(C) The Author(s) 2019

\begin{abstract}
Rationale Patients with obsessive-compulsive disorder (OCD) have been found to show exaggerated error responses and prediction error learning signals in a variety of EEG and fMRI tasks, with data converging on the anterior cingulate cortex as a key locus of dysfunction. Considerable evidence has linked prediction error processing to dopaminergic function.

Objective In this study, we investigate potential dopaminergic dysfunction during reward processing in the context of OCD. Methods We studied OCD patients $(n=18)$ and controls $(n=18)$ whilst they learned probabilistic associations between abstract stimuli and monetary rewards in the fMRI scanner involving administration (on separate visits) of a dopamine receptor agonist, pramipexole $0.5 \mathrm{mg}$; a dopamine receptor antagonist, amisulpride $400 \mathrm{mg}$; and placebo. We fitted a Q-learning computational model to fMRI prediction error responses; group differences were examined in anterior cingulate and nucleus accumbens regions of interest. Results There were no significant group, drug, or interaction effects in the number of correct choices; computational modeling suggested a marginally significant difference in learning rates between groups $\left(p=0.089\right.$, partial $\left.\eta^{2}=0.1\right)$. In the imaging results, there was a significant interaction of group by drug $\left(p=0.013\right.$, partial $\left.\eta^{2}=0.13\right)$. OCD patients showed abnormally strong cingulate signaling of prediction errors during omission of an expected reward, with unexpected reduction by both pramipexole and amisulpride $\left(p=0.014\right.$, partial $\eta^{2}=0.26,1-\beta$ error probability $\left.=0.94\right)$. Exaggerated cingulate prediction error signaling to omitted reward in placebo was related to trait subjective difficulty in self-regulating behavior in OCD.

Conclusions Our data support cingulate dysfunction during reward processing in OCD, and bidirectional remediation by dopaminergic modulation, suggesting that exaggerated cingulate error signals in OCD may be of dopaminergic origin. The results help to illuminate the mechanisms through which dopamine receptor antagonists achieve therapeutic benefit in OCD. Further research is needed to disentangle the different functions of dopamine receptor agonists and antagonists during bidirectional modulation of cingulate activation.
\end{abstract}

Keywords Obsessive-compulsive disorder $\cdot$ Amisulpride $\cdot$ Pramipexole $\cdot$ Reward learning $\cdot$ Prediction error $\cdot$ Anterior cingulate $\cdot$ Nucleus accumbens · Computational model

Graham K. Murray and Franziska Knolle contributed equally to this work.

Electronic supplementary material The online version of this article (https://doi.org/10.1007/s00213-019-05292-2) contains supplementary material, which is available to authorized users.

Graham K. Murray

gm285@cam.ac.uk

Franziska Knolle

fk291@cam.ac.uk

1 Department of Psychiatry, University of Cambridge, $18 \mathrm{~b}$

Trumpington Road, Cambridge CB2 8AH, UK

2 Behavioural and Clinical Neuroscience Institute, University of Cambridge, Cambridge CB2 1TN, UK
3 Cambridgeshire and Peterborough NHS Foundation Trust, Cambridge CB21 5EF, UK

4 Department of Psychology, University of Cambridge, Cambridge CB2 1TN, UK

5 European Bioinformatics Institute, Cambridge CB10 1SD, UK

6 GlaxoSmithKline, Immuno-Inflammation Therapeutic Area Unit, Stevenage, UK

7 Department of Psychiatry, Queen Elizabeth II Hospital, Welwyn Garden City, UK 


\section{Introduction}

Obsessive-compulsive disorder (OCD) has been associated with deficits in learning and decision making (Gillan and Robbins 2014; Nielen et al. 2009). OCD patients were found to show reduced response control in punishment trials, with impulsivity being correlated with severity of symptoms (Morein-Zamir et al. 2013), and abnormal error responses in a variety of response conflict and error monitoring tasks using EEG (Gillan et al. 2017; Mathews et al. 2012), suggesting overactivity of the performance monitoring system involving the anterior cingulate cortex (Hammer et al. 2009).

Neural mechanisms underlying decision making and learning involve fronto-striatal networks generating reward prediction errors which are highly sensitive to dopaminergic modulation (Frank et al. 2004; Kehagia et al. 2010; Schultz and Dickinson 2000). Extensive evidence from studies in experimental animals has demonstrated that dopamine neurons show increased rates of firing to unexpected or better than expected rewards (positive prediction error), and a reduction in firing rate during omission of expected rewards (negative prediction error) (Schultz et al. 1997). Studies in healthy human volunteers have shown dopaminergic modulation of the brain response to a reward prediction error response using fMRI (Bernacer et al. 2013; Eisenegger et al. 2014; Pessiglione et al. 2006). Evidence from human molecular imaging studies of patients with OCD suggests a possible role for dopaminergic pathology in OCD (Denys et al. 2004; Hesse et al. 2005; Moresco et al. 2007; Olver et al. 2009, 2010; Perani et al. 2008; Sesia et al. 2013; Van Der Wee et al. 2004). Whilst dopaminergic medications are not first-line treatments for OCD, dopamine receptor antagonists are often used to augment first-line treatment in refractory cases (Hirschtritt et al. 2017) and the efficacy of this strategy is confirmed by meta-analyses (Dold et al. 2015; Veale et al. 2014).

Notably, the nucleus accumbens (part of the ventral striatum) receives dense dopaminergic projections and is a target for deep brain stimulation in OCD (Denys et al. 2010). A prior study demonstrated abnormal reward processing in the nucleus accumbens in OCD (Figee et al. 2011): it reported attenuated activations for reward anticipation but did not examine learning or prediction error signaling. The nucleus accumbens has notably been associated with coding prediction error in healthy controls (Abler et al. 2006; O'doherty et al. 2004; Pagnoni et al. 2002; Rodriguez et al. 2006). The concept of negative prediction error is closely related to that of error monitoring, which is reliably abnormal in OCD, although the two processes are not identical. Both processes involve evaluating worse than expected outcome, motivating the study of prediction error signaling in OCD. Recently, Hauser et al. (2017) reported increased brain signals in response to reward prediction errors in the anterior cingulate cortex and the putamen, indicating an overactivation of the monitoring system. Furthermore, dopamine-dependent activation in the anterior cingulate has also been associated with increased error awareness and performance monitoring (Hester et al. 2012; Pauls et al. 2012). Reward prediction error processing is closely linked to the dopaminergic system (Schultz et al. 1997). A plausible hypothesis unifying (I) the known role of dopamine in signaling reward prediction errors, (II) the existing evidence of dopaminergic pathology in OCD, and (III) evidence of abnormal error processing in OCD is that OCD patients may exhibit abnormal processing of reward prediction errors, especially during omission of expected reward linked to abnormal dopaminergic function.

To our knowledge, no study has yet examined a direct modulation of the dopaminergic system on a reward prediction error task in OCD. In the current experiment, we therefore compared the effect of dopaminergic drug treatments on prediction error signaling in OCD. We recruited a group of patients with OCD and a group of healthy volunteers as controls, and examined brain responses during studies of reinforcement learning in an fMRI scanner after administration of a dopamine $D_{2 / 3}$ receptor agonist, pramipexole, or a dopamine $D_{2 / 3}$ receptor antagonist, amisulpride, or a placebo. We hypothesized that fronto-striatal reward prediction error signaling in OCD would be exaggerated under placebo; given the evidence for dopamine receptor antagonism add-on treatment for OCD, we hypothesized that this abnormality would be normalized by amisulpride but not by pramipexole, which has been shown in some cases to worsen compulsivity (Kolla et al. 2010). Our hypotheses were principally focused on two brain regions of particular interest: we predicted that dopaminergic abnormalities in anterior cingulate reward prediction errors would be prominent in OCD during processing of omitted rewards, as this is analogous to the enhanced cingulate error signaling suggested by error-related negativity studies in OCD (Gillan et al. 2017). A secondary hypothesis was that, in the nucleus accumbens (Figee et al. 2011), there would be dopaminergic reward prediction error abnormalities in OCD (during reward receipt and/or omission).

\section{Methods}

\section{Participants}

Thirty-six right-handed volunteers participated in this study (Table 1): 18 individuals satisfying DSM-IV-TR criteria for OCD, and 18 healthy controls. The groups were matched for age, gender, IQ, and years of education. Prior to study enrollment, all participants had a satisfactory medical review and were screened for any other current Axis I psychiatric disorder using the Structured Clinical Interview for the DSM-IV-TR Axis I psychiatric disorders. OCD patients were recruited 
from a specialist OCD clinic and through independent charities, and most were taking serotonin selective reuptake inhibitor (SSRI) medication (see Supplementary Material for details). Participants were excluded based on any other Axis I psychiatric disorders. All volunteers provided written informed consent. The study was approved by the Cambridge Research Ethics Committee.

\section{Pharmacological interventions}

Participants attended for three in-unit assessments in which treatments were administered by mouth. On one visit, they received $0.5 \mathrm{mg}$ pramipexole, a selective agonist at the dopamine $\mathrm{D}_{2 / 3}$ receptors; on another visit, $400 \mathrm{mg}$ amisulpride, a selective antagonist at the dopamine $\mathrm{D}_{2 / 3}$ receptors, and on another visit, a placebo. Drug administration was conducted in a double-blind, placebo-controlled fashion counterbalanced for drug/visit order. Each dosing of drug/placebo was administrated $60 \mathrm{~min}$ prior to scanning to assure peak plasma levels for both drugs during scanning. The time point of drug administration was based on pharmacokinetic data for both drugs (Coukell et al. 1996; Rosenzweig et al. 2002; Wright et al. 1997).

Patients' eligibility to proceed with the scanning was assured by ECG monitoring. We initially administered a single oral dose of $1.5 \mathrm{mg}$ of pramipexole to the first three healthy volunteers. However, this dose of pramipexole was poorly tolerated; the three healthy volunteers were unable to perform the tasks at this treatment session because of nausea, vomiting, sweating, and tiredness. Subsequently, the dose of pramipexole was reduced to $0.5 \mathrm{mg}$ orally for all participants (i.e., for 18 controls, including the three volunteers who did not tolerate the higher dose). All participants were also administered a total of $30 \mathrm{mg}$ of domperidone orally for each treatment session to prevent emetic effects of dopamine receptor agonism. The administration was split up over three time points. Ten milligrams of domperidone was to be taken $12 \mathrm{~h}$ and $2 \mathrm{~h}$ before arrival, and further $10 \mathrm{mg}$ of domperidone was administered together with the study medication.

Subjective drug effects were assessed at two time points, 1 and $1.5 \mathrm{~h}$ after drug administration using the Bond-Lader Visual Analogue Scale (BOND and LADER 1974). The time points referred to assessment immediately before and after fMRI scanning. At this time points, blood samples were taken from the participants, to assess plasma-levels and prolactin concentration.

\section{fMRI task}

During the fMRI scan, subjects carried out a probabilistic learning task that required making choices to maximize wins and minimize losses (Fig. 1), adapted from previous similar tasks (Bernacer et al. 2013; Ermakova et al. 2018; O'doherty et al. 2004; Pessiglione et al. 2006). In each trial, one of three possible pairs of abstract pictures was randomly presented: rewarding, punishing, or neutral (40 trials of each valence). For each trial, the subject used a button push to indicate a choice of picture. When viewing the potentially rewarding pair, selection of one of the pictures led to a financial win with a $70 \%$ probability and of a no-change outcome with $30 \%$ probability, whereas the selection of the other picture led to gain with only $30 \%$ probability. The potentially punishing pair led to a financial loss on $70 \%$ and $30 \%$ trials depending on stimulus choice, and the neutral pair led to no change. We focus in this report on how participants learned about rewards; hence, we report fMRI results from the reward trials only. Details of behavior on other trial types are reported in the Supplementary Material.

\section{Behavioral analysis}

We used a repeated measures analysis of variance (ANOVA) to investigate the group differences (controls, OCD; between subjects' variable) and effect of drug (placebo, pramipexole, amisulpride; within subjects' variable) in stimuli choices and reaction times. We were interested in the proportion with which the subjects gave a "correct" response and whether this performance was dependent on trial types and drugs. Stimulus selection was considered correct when choosing the stimulus with the high probability of leading to winning of 50 pence in the reward trial (regardless of whether or not the participant was rewarded on that particular trial), the picture with the high probability of receiving a neutral feedback picture in neutral trials, and stimulus with the high probability of leading to no punishment. In the neutral trials, the assignment of "correctness" is arbitrary but assigning one stimulus in each category to be the correct stimulus allows examination of to what extent response patterns differed across trial types.

Computational learning model parameters (see below) were analyzed in a repeated measures ANOVA with group as a between-subjects' variable and drug as a within-subjects' variable.

\section{Computational modeling of learning}

We estimated the positive and negative reward prediction error value for each trial, based on a basic Q learning algorithm (Murray et al. 2008; Pessiglione et al. 2006), that involves modeling learning rates (alpha) and "explorationexploitation" parameters (betas). Beta, also understood as the inverse temperature, balances between random sampling or exploration of actions, and the exploitation of current knowledge (see the Supplementary Material for the detailed description of the computational modeling methods). 
Table 1 Subject characteristics. Mean scores (SD) are shown for continuously distributed variables according to diagnostic group

\begin{tabular}{|c|c|c|c|c|c|}
\hline & \multirow[b]{2}{*}{ Controls } & \multirow[b]{2}{*}{ OCD } & \multicolumn{3}{|c|}{ Statistical results } \\
\hline & & & $t$ & df & $p$ \\
\hline Age (years) & $32.1(6.5)$ & $35.6(10.1)$ & 0.95 & 34 & 0.35 \\
\hline Gender (male:female) & $15: 3$ & $11: 7$ & 1.49 & 34 & 0.15 \\
\hline Height $(\mathrm{cm})$ & $177.0(9.1)$ & $170.4(8.3)$ & 2.25 & 33 & 0.03 \\
\hline Weight (kg) & $79.2(12.6)$ & $80.4(12.7)$ & 0.26 & 32 & 0.80 \\
\hline BMI & $25.1(2.9)$ & $27.8(0.6)$ & 1.97 & 32 & 0.06 \\
\hline Verbal IQ (NART) & $108.2(6.1)$ & $107.8(9.2)$ & 0.22 & 34 & 0.83 \\
\hline Years of education & $12.5(1.8)$ & $12.4(2.1)$ & 0.26 & 34 & 0.80 \\
\hline Self-regulation scale (SRQ total score at baseline) & $225.7(16.2)$ & $199.9(21.6)$ & 4.02 & 33 & $<0.001$ \\
\hline Searching for options & $31.9(4.0)$ & $29.7(5.0)$ & 1.43 & 33 & 0.17 \\
\hline Implementing a plan & $34.7(3.0)$ & $26.9(5.1)$ & 5.56 & 33 & $<0.001$ \\
\hline Triggering change & $31.1(2.1)$ & $28.5(3.1)$ & 2.92 & 33 & 0.006 \\
\hline Age of onset (years) of OCD) & - & $17.8(10.9)$ & & & \\
\hline Severity of OCD (Y-BOCS score at baseline) & & $24.1(6.8)$ & & & \\
\hline
\end{tabular}

\section{MRI analysis}

MRI acquisition is described in the Supplementary Material. Data were initially analyzed a voxelwise fashion using FSL software (Jenkinson et al. 2012). Data were analyzed using the FSL tool, FEAT (FMRI Expert Analysis Tool), using the following steps. EPI images were realigned, motion corrected, and spatially smoothed with a Gaussian kernel of $6 \mathrm{~mm}$ (full-width half-maximum). The time series in each session was high-pass filtered (60 s cut-off), and the images were registered to the structural MPRAGE scan obtained from the corresponding subject (having first removed the skull using the brain extraction tool BET) and finally transformed into standard space using the MNI template.

In the analysis of fMRI data, eight explanatory variables (EVs), along with their temporal derivatives (used instead of slice timing correction), were defined in our model as follows: (1) reward expected value; (2) positive reward prediction error; (3) negative reward prediction error; (4) punishment expected value; (5) positive punishment PE; (6) negative punishment PE; (7) feedback presentation; (8) cue presentation. FSL requires all events to be set with non-zero duration: we set all to be $2 \mathrm{~s}$. EVs $1-6$ were based on the trial by trial computational model estimates of reward cue values and prediction errors. The timings of EVs 1 and 4 correspond to cue presentation. EVs 2, 3, 5 and 6 correspond to the times of delivery of reward or omission of expected reward. EV2 represents reward prediction error when a reward is received (i.e., positive prediction error), and EV3 represents the prediction error when an expected reward is omitted (negative prediction error). A positive EV3 parameter estimate, therefore, reflects lower BOLD values during omission of predicted rewards. Positive and negative reward prediction error activity were considered separately, as prior research in OCD has focused on brain correlates of errors using tasks where an error is usually accompanied by negative feedback or represents some kind of mistake, which is more analogous to negative prediction error than positive prediction error. In the present analysis, we focused on EV2 and EV3, the positive and negative reward prediction errors. We computed separate +1 contrasts on EV2, and on EV3 at the single subject level (contrast of parameter estimates, or COPEs). We defined two regions of interest (ROIs: see below and Supplementary Material) and extracted average COPE values from each participant in these ROIs, in order to subject these to ANOVA in the statistics package SPSS. Before ANOVA was performed, we examined the distributions of the COPE values and excluded outliers more than three standard deviations away from the mean of all the samples pooled (Howell 2009). Although, psychologically, punishment learning is of considerable interest in OCD, the neurochemical basis of punishment prediction error signals is much less clear than those of reward prediction errors, with prior studies (Pessiglione et al. 2006) failing to find evidence of dopaminergic modulation of punishment prediction error learning signals. For this reason, although our paradigm included punishment learning, we did not proceed to formulate or test hypotheses regarding punishment brain signals.

In this study, we were interested in the anterior cingulate cortex, using a sphere, $14 \mathrm{~mm}$, centered at $x=0, y=42, z=18$, based on (Hauser et al. 2017), and the nucleus accumbens, as defined as in the Harvard-Oxford-subcortical atlas supplied with FSL, during prediction error processing.

\section{Relation to behavioral scales}

We determined relationships between anterior cingulate and accumbens prediction error COPE values in placebo and behavioral scales using nonparametric correlations (Spearman's rho). We examined total OCD symptoms (in patients only) as 
a
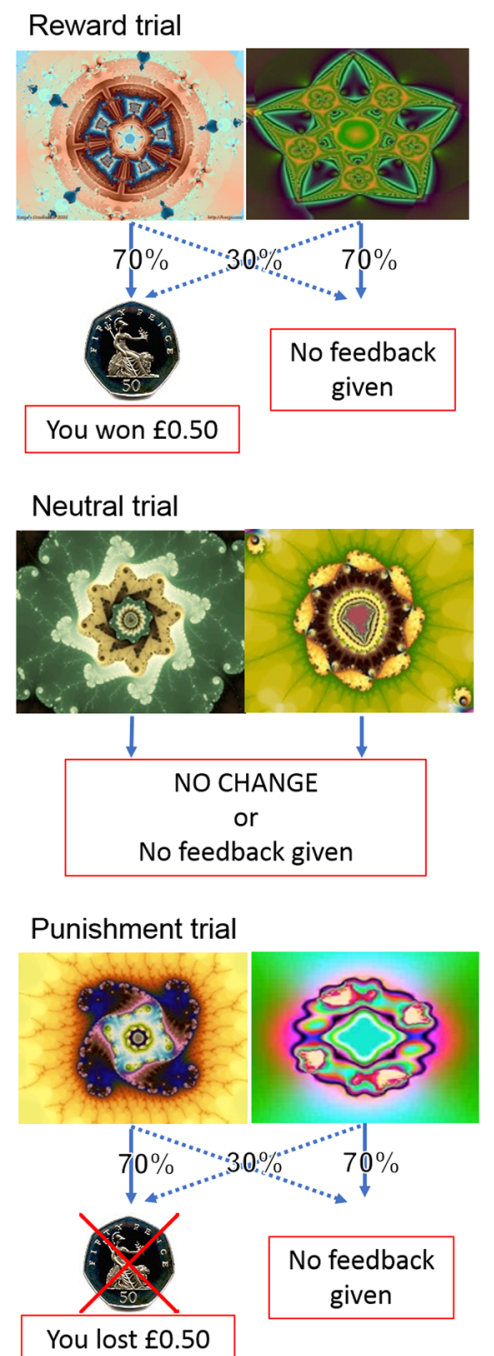

Fig. 1 Panel a presents the three different trial types and feedback probabilities. Panel b presents the experimental task, including trial timing. With cue presentation, participants have a maximum of $2 \mathrm{~s}$ to

measured by the Y-BOCS scale (Goodman et al. 1989) and a subjective assessment of goal-directed behavior/cognitive control (in both groups) using the self-regulation questionnaire (SRQ), which assesses the trait ability to develop, implement, and flexibly maintain planned behavior in order to achieve one's goals (Brown et al. 1999). It contains items for receiving relevant information, evaluation, triggering change, searching for option, formulating a plan, implementing a plan, and assessing effectiveness.

\section{Results}

\section{Discrimination learning}

Independent of drug, participants learned to choose the picture that most often led to winning 50p (high-probability stimulus:

b

$\max 2$
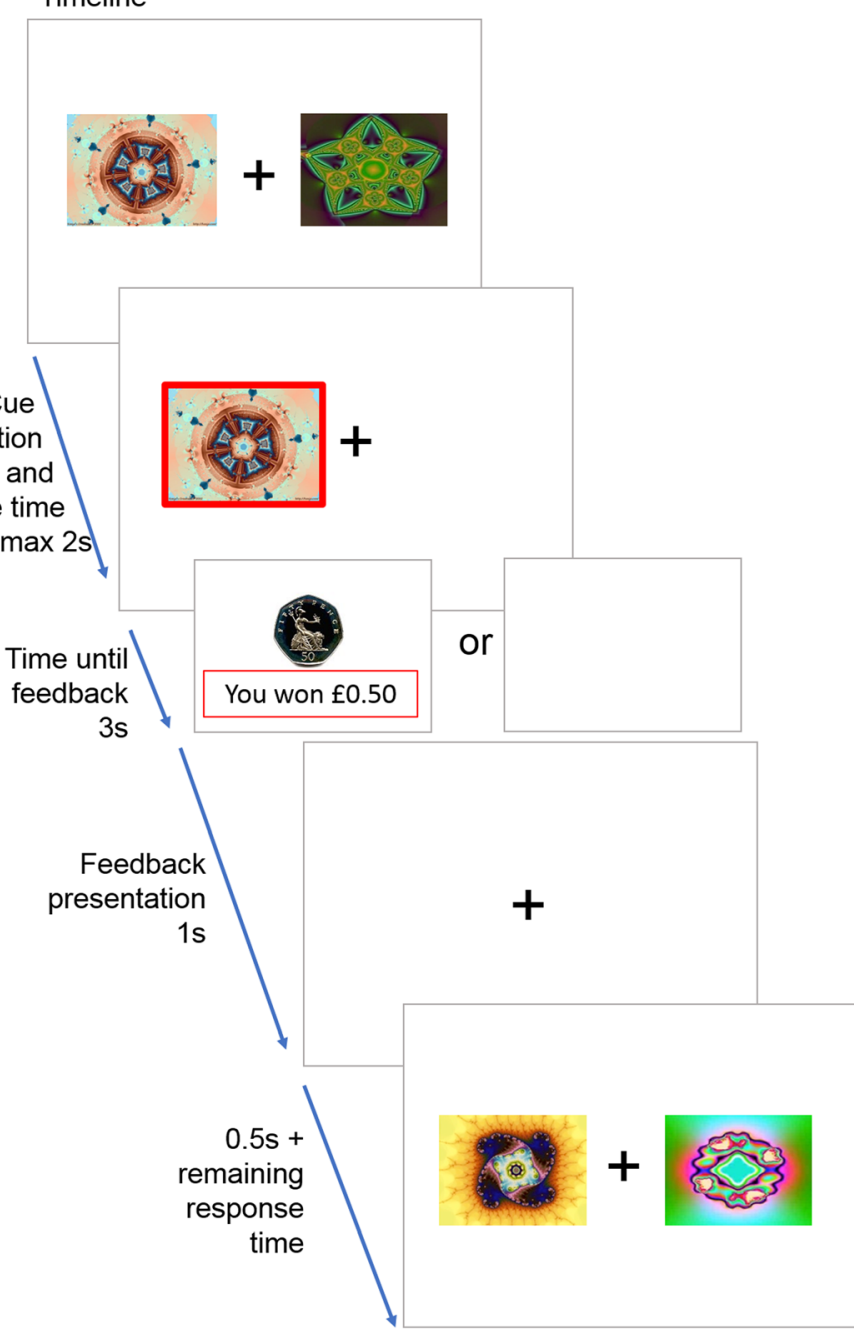

make a decision. The chosen stimulus is circled in red and presented for $3 \mathrm{~s}$. Feedback or no feedback is presented for $1 \mathrm{~s}$, which is followed by a fixation cross for a minimum of $0.5 \mathrm{~s}(0.5 \mathrm{~s}+(2 \mathrm{~s}$-reaction time $))$

correct response) in the majority of reward trials (mean percentage of correct choices for controls $83.58 \%( \pm 26.3)$ and patients $72.45 \%( \pm 34.9)$ ); there was a tendency for OCD patients to make more errors across all treatment conditions (Fig. 2) (marginal effect: $F=3.5$, dfn $=1$, dfe $=34, p=$ 0.070 , partial $\left.\eta^{2}=0.09\right)$, but no effect of drug $(p=0.39)$ or group X drug interaction $(p=0.86)$. (See Supplementary Material for learning curves for reward trials, and description of other trial types.)

\section{Reaction time results}

There was a significant main effect of $\operatorname{drug}(F=5.63, \mathrm{dfn}=2$, dfe $=68, p=0.005$, partial $\eta^{2}=0.14$ ), but no effect of group $(p=0.62)$ or an interaction $(p=0.70)$. Bonferroni corrected post hoc tests revealed that participants independently of group reacted to the reward trials significantly slower under 
pramipexole $(845.29 \mathrm{~ms} \pm 29.22)$ compared with placebo (770.93 ms $\pm 23.66 ; p=0.016)$, or amisulpride (757.86 $\mathrm{ms} \pm$ 26.41; $p=0.002$ ) (see Supplementary Material Results and Table 1 for more details).

\section{Computational modeling parameters}

For the learning rate, $\alpha$, we found a marginal effect of group ( $F=3.07, \mathrm{dfn}=1, \mathrm{dfe}=34, p=0.089$, partial $\left.\eta^{2}=0.1\right)$, but no effect of drug $(p=0.94)$ or an interaction $(p=0.31)$. The marginally significant group effect suggests that controls, independent of drug, have a higher learning rate (mean $\alpha=0.27$, standard error 0.02) than OCD patients (mean $\alpha=0.22$, standard error 0.02). For $\beta$, there were no differences across groups $(p=0.82)$, drug $(p=0.1)$ or an interaction $(p=0.78)$. There was no effect of drug $(p=0.38)$, group $(p=0.22)$, or interaction $(p=0.54)$ on model fit.

\section{Imaging results, pooled data ("main effect of task")}

Results from pooled data, collapsing over group and drug conditions, are presented in Supplementary Material.

\section{Imaging results: anterior cingulate cortex, negative prediction error}

The mean COPE values for negative prediction error in the anterior cingulate cortex were extracted to enable a region of interest analysis in accordance with our hypothesis. Data from three OCD patients were excluded because, for at least one of the sessions, the mean COPE value from the ROI was over three standard deviations from the mean of the entire sample. Following repeated measure ANOVA, there were no main effects of group $(p=0.88)$ or drug $(p=0.44)$. However, there was a significant interaction of group by drug $(F=4.65, \mathrm{dfn}=$ 1,2 , dfe $=30,62, p=0.013$, partial $\eta^{2}=0.13,1-\beta$ error probability $=0.7)$. Within each group, we then conducted a Bonferroni corrected post hoc test analyzing the effect of the drug with a repeated measures ANOVA. In controls, the drug intervention did not influence negative reward prediction error processing, as shown by a non-significant drug effect ( $p=$ $0.16)$. In the OCD patients, however, there was a significant drug effect $\left(F=5.01\right.$, dfn $=2$, dfe $=28, p=0.014$, partial $\eta^{2}=$ $0.26,1-\beta$ error probability $=0.94)$. On further analysis within the patient group, amisulpride (mean $=-40.03, \mathrm{SD}=157.35$ ) as well as pramipexole (mean $=-20.83, \mathrm{SD}=133.09$ ) led to significant differences compared with placebo but not between themselves $($ mean $=128.56, \mathrm{SD}=189.54)$ : placebo $>$ amisulpride, $p=0.030$; placebo $>$ pramipexole, $p=0.017$; amisulpride $>$ pramipexole, $p=0.70$.

Furthermore, we applied a univariate ANOVA to each drug intervention separately (Fig. 3). For placebo, there was a significant effect for group $(F=8.84, \mathrm{dfn}=1, \mathrm{dfe}=31, p=$
0.006 , partial $\eta^{2}=0.22,1-\beta$ error probability $=0.91$ ), showing that the COPE values in the controls (mean $=-42.21, \mathrm{SD}=$ $140.24)$ were lower than in OCD $($ mean $=128.57, \mathrm{SD}=$ 189.54). For the other two interventions, no significant differences were found between groups (amisulpride: controls $($ mean $=15.12, \mathrm{SD}=243.59)>\mathrm{OCD}($ mean $=-40.03, \mathrm{SD}=$ $157.35), p=0.46$; pramipexole: controls (mean $=117.4, \mathrm{SD}=$ $362.97)>\mathrm{OCD}($ mean $=-20.83, \mathrm{SD}=133.09), p=0.17)$.

\section{Imaging results: anterior cingulate cortex, positive prediction error}

There were no significant group, drug, or interaction effects for positive prediction error in the anterior cingulate cortex in a repeated measure ANOVA.

\section{Imaging results: nucleus accumbens, negative prediction error}

There were no significant group or drug or interaction effects on negative prediction error COPE values in the nucleus accumbens.

\section{Imaging results: nucleus accumbens, positive prediction error}

The mean COPE values for positive prediction error in the nucleus accumbens were extracted to enable a region of interest analysis in accordance with our hypothesis. Data from three controls and one OCD patient were excluded as, on at least one of the different treatment sessions, the mean COPE values from the ROI were over three standard deviations from the mean of the entire sample. Following repeated measure ANOVA, there was a significant main effect of group $(F=$ $11.82, \mathrm{dfn}=1, \mathrm{dfe}=30, p=0.002$, partial $\eta^{2}=0.28,1-\beta$ error probability $=0.96)$, but no significant effect for drug $(p=$ $0.45)$ or interaction of group by drug $(p=0.54)$.

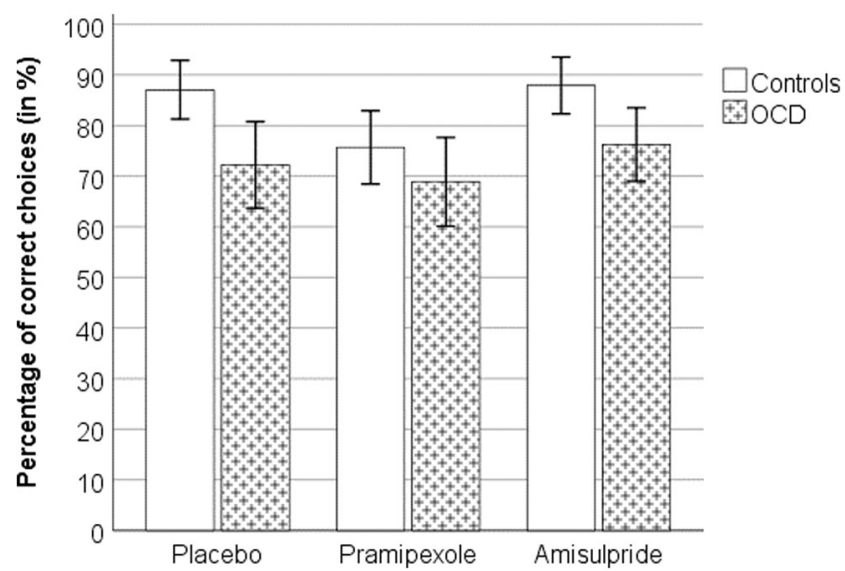

Fig. 2 Choice performance (mean \pm 1 SEM) during reward trials under different drugs, separated by group 
We then applied a univariate ANOVA to each drug intervention separately (Fig. 4). For placebo, there was a significant effect for group $(F=5.11, \mathrm{dfn}=1$, dfe $=30 p=0.031$, partial $\eta^{2}=0.15,1-\beta$ error probability $=0.75$ ), showing that the COPE values in the controls (mean $=32.28, \mathrm{SD}=120.46$ ) were significantly less than in the OCD group (mean = $125.74, \mathrm{SD}=113.32$ ). For the amisulpride intervention, no significant differences were found between groups (amisulpride: controls $($ mean $=89.15, \mathrm{SD}=125.77)>\mathrm{OCD}$ $($ mean $=110.81, \mathrm{SD}=122.66) ; p=0.63)$. For the pramipexole intervention, we found a marginal effect $(F=2.90, \mathrm{dfn}=1$, $\mathrm{dfe}=30, p=0.099$, partial $\eta^{2}=0.09$ ) with controls (mean = 23.36, $\mathrm{SD}=124.58)$ showing less activity compared with OCD patients $($ mean $=93.21, \mathrm{SD}=107.35)$.

We also conducted a Bonferroni corrected post hoc test analyzing the effect of the drug within each group using repeated measure ANOVA. Neither in controls nor in OCD patients did the drug intervention influence reward positive prediction error processing (controls: $p=0.99$; OCD patients: $p=0.60$ ).

\section{SSRI treatment-medication interaction}

In order to control for a potential interaction of the patients' individual routine medication and the study drug treatment on reward prediction error signaling, we created a dichotomized medication variable (high dose or not high dose, the latter including two medication-free patients) and used this variable as a between-subject factor in a repeated measure ANOVA on the three treatment types (i.e., placebo, pramipexole, amisulpiride). We found a significant treatment effect on anterior cingulate cortex negative prediction error COPE values $(F=5.42$, dfd $=$ 2 , dfe $=6, p=0.011$ ), as in our analysis above without the medication factor, but no SSRI medication dose effect $(p=$ $0.8)$ or interaction $(p=0.3)$. In the nucleus accumbens, we did not find any significant effect of high SSRI dose or interaction on positive prediction error COPE values (all $p>0.1$ ).

\section{Correlations with behavioral scales}

There was no correlation between placebo cingulate negative prediction error COPE values and OCD symptoms as measured by Y-BOCS score (rho $=-0.2, p=0.4)$. However, there was a significant negative correlation between the cingulate negative prediction error signal and trait self-regulation as measured by the SRQ (rho $=-0.7, p=0.002$, including outliers; rho $=-0.75, p=0.002$, excluding outliers), such that patients who reported more difficulty with self-regulation also demonstrated exaggerated negative error signals in the anterior cingulate (Fig. 5). On analyses of subscales, the overall association was mainly driven by associations with subscales for "triggering change," "searching for options," and "implementing a plan" (all $p<0.005$ ).

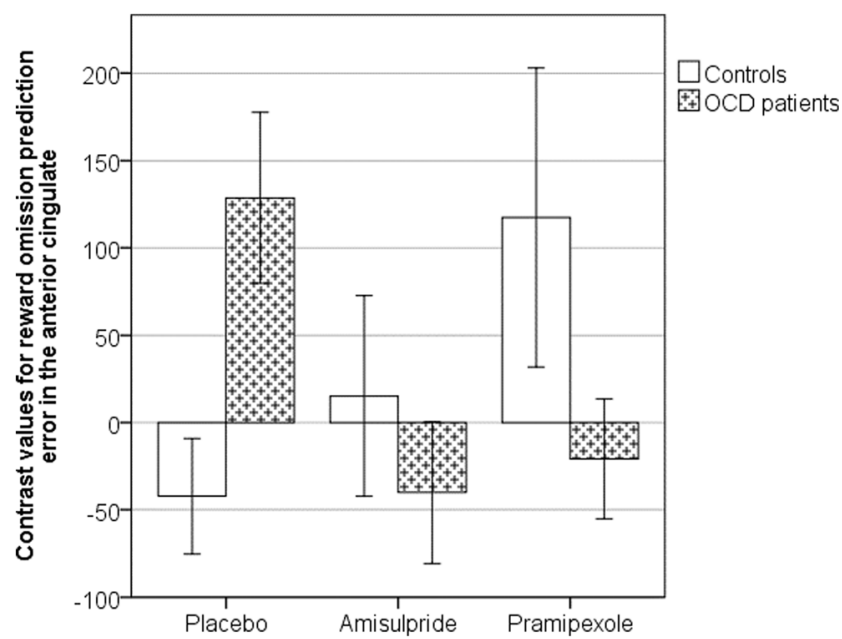

Fig. 3 Bar chart showing mean negative reward prediction error contrast value ( $\pm 1 \mathrm{SEM})$ extracted from the anterior cingulate cortex at the time of reward omission. A higher contrast value indicates that the BOLD signal was positively correlated with negative prediction error. OCD patients were significantly different from controls following placebo, but both groups behave similarly following amisulpride or pramipexole

There were no correlations between placebo COPE values in the nucleus accumbens and rating scales (Y-BOCS, selfregulation including subscales, all $p>0.5$ ).

\section{Discussion}

In this pharmacological fMRI study of reinforcement learning, we found that OCD patients exhibited abnormally increased signaling of prediction errors to omitted rewards in the anterior cingulate cortex under placebo treatment, which was reduced by acute dopaminergic drug treatment using either pramipexole or amisulpride. This bidirectional reduction was unexpected and requires further analysis in future research. Furthermore, we found that OCD patients also showed abnormally increased activity associated with positive prediction errors in the nucleus accumbens in placebo, which was, however, unaffected by dopaminergic drug treatment.

In OCD, during placebo, the anterior cingulate representation of negative prediction error (prediction error during omitted rewards) was significantly greater than in controls. In controls, we did not detect a clear cingulate prediction error signal during omitted rewards. It is possible that our paradigm was not sufficiently sensitive to detect a negative prediction error signal in the control group, although it was clearly sufficiently sensitive to detect the pronounced signal in OCD. The lack of clear prediction errors in controls may have resulted from our procedure containing only 40 reward pair stimuli trials; for comparison, other prior prediction error fMRI studies have included larger numbers of potentially rewarding trials, such as 80 (Murray et al. 2008) or 90 (Pessiglione et al. 2006). Pronounced anterior cingulate prediction error signals are 
consistent with a recent study reporting stronger fronto-striatal reward prediction error signals in OCD, also using an fMRI reinforcement learning paradigm (Hauser et al. 2017). Furthermore, exaggerated responses to errors are a consistent finding in OCD, with a number of prior studies showing an overactivation of frontal responses to error processing in OCD in both fMRI research (Stern et al. 2011; Ursu et al. 2003) and EEG error-related negativity studies (Gillan et al. 2017; Mathews et al. 2012).

One previous study of reward anticipation (not learning) showed nucleus accumbens dysfunction in OCD (Figee et al. 2011). Here, we report an overactivation in the nucleus accumbens responses to positive prediction errors in OCD, consistent with prior data showing overactivation in OCD across error tasks and brain regions (Stern et al. 2011; Ursu et al. 2003) and in line with results from Hauser and colleagues (Hauser et al. 2017), who showed excessive striatal reward prediction errors in OCD during reversal learning.

We found no significant correlations between anterior cingulate prediction error signals and a general OCD symptom score; this lack of association is in accordance with the results from the only other fMRI study of reward prediction error in OCD (Hauser et al. 2017). However, we did demonstrate an association between anterior cingulate negative prediction error signal with self-perceived "self-regulation": driven by associations with subcomponents of difficulty in triggering behavioral change, searching for options, and implementing a plan. This result may be relevant to much recent data suggesting an impairment of goal-directed behavior in OCD and highlights the importance of cingulate dysfunction in this impairment (Giele et al. 2016; Gillan and Robbins 2014). Our data suggest that the self-regulation questionnaire may reflect underlying anterior cingulate function in OCD. We are not

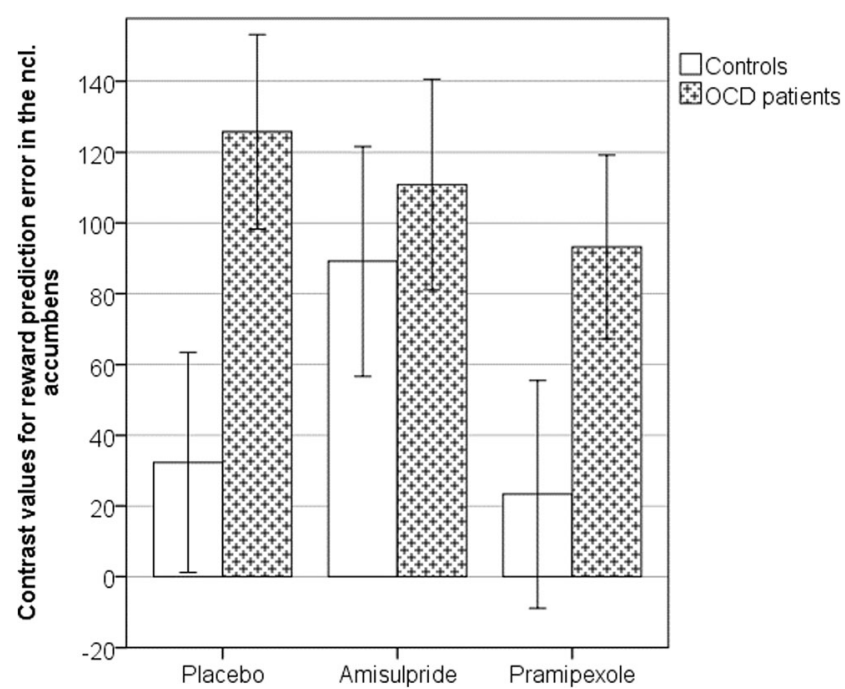

Fig. 4 Bar chart showing mean reward prediction error contrast values ( \pm 1 SEM) extracted from the nucleus accumbens. OCD patients were significantly different from controls following placebo, but both groups behave similarly following amisulpride or pramipexole aware of any clinical trials for OCD that have used this scale as an outcome measure; however, our results suggest that including a questionnaire measure of goal-directed behavioral control, such as the SRQ, might be useful in future clinical trials of interventions targeting cingulate mediated deficits in OCD.

Although the literature provides consistent evidence linking error processing to dopaminergic function (Schultz et al. 1997) - a system apparently impaired in OCD (Denys et al. 2004; Hesse et al. 2005; Moresco et al. 2007; Olver et al. 2009, 2010; Perani et al. 2008; Sesia et al. 2013; Van Der Wee et al. 2004) —our study extends prior work by directly showing the effect of dopaminergic modulation on brain prediction error responses in OCD. Overactivation of the anterior cingulate cortex in response to negative reward prediction errors in OCD was remediated by amisulpride, the selective $\mathrm{D}_{2 / 3}$ receptor antagonist, as hypothesized. However, the D2/3 agonist pramipexole unexpectedly had similar, rather than opposite, effects.

Nevertheless, previous studies have shown that both drugs can exert bidirectional effects on reward prediction errorassociated responses. Thus, amisulpride or similar $\mathrm{D}_{2 / 3}$ receptor antagonists can either enhance (Jocham et al. 2011) or depress (Abler et al. 2007) reward-related brain activity. Furthermore, dopamine agonist-like medications have also been shown to be capable of enhancing (Pessiglione et al. 2006) or attenuating (Bernacer et al. 2013) reward prediction error-associated responses, with the directionality of the effect being dependent on the particular task, agonist, and dose employed. Two obvious accounts of this pattern of effects are that either (I) both agents in our study reduce prediction errors by operating at opposite sides of a hypothetical inverted

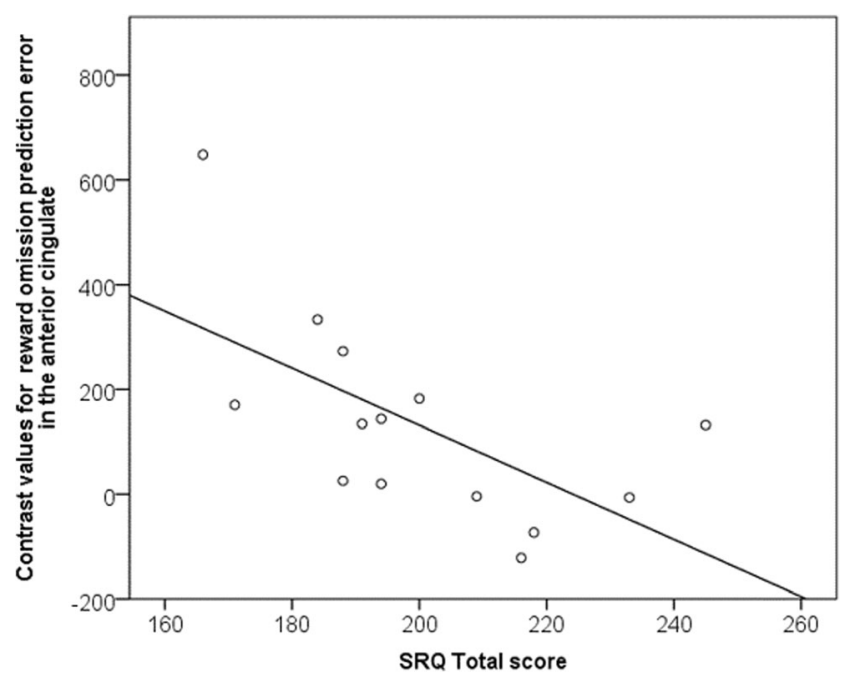

Fig. 5 Significant correlation between self-regulation and negative prediction error activation in the anterior cingulate region of interest for OCD patients (rho $=-0.7, p=0.002)$ : stronger representation of prediction error during reward omission related to weaker self-regulation. SRQ - self-regulation questionnaire 
U-shaped function that determines the net effect of dopaminergic modulation on the computation of prediction error or (II) actions at inhibitory presynaptic receptors, at the level of the ventral tegmental area (Grace and Bunney 1983) and/or via terminal autoreceptors (e.g., (Horst et al. 2019)), may be implicated for one or other drugs that are opposite in sign to their postsynaptic effects. As the dosage of amisulpride in our study is between a high and a low dose, the latter account is further supported by the finding that low doses of amisulpride in animal studies are associated with a facilitation of behaviors dependent on presynaptic dopamine receptor activation, whereas higher doses were found to decrease behaviors associated with postsynaptic receptor activation (Perrault et al. 1997). Such an action can only be assessed by determining an entire dose-response curve, and it is an obvious limitation of the present data that this was not feasible in this study of human participants. In particular, it is possible that the low dose $(0.5 \mathrm{mg})$ of pramipexole employed in our study may have resulted primarily in presynaptic effects at presynaptic D2 autoreceptors which have higher affinity for dopamine than postsynaptic heteroreceptors (Ford 2014). It is also possible that the degree to which our study drugs act pre- versus postsynaptically may depend on dopamine receptor availability, presynaptic action being favored when receptor availability is low. We note prior evidence of low dopamine receptor availability in OCD (Denys et al. 2004), which is likely to contribute to differential drug effects between OCD patients and controls and is consistent with the observed effects of these dopaminergic agents. We initially intended to use a higher dose - $1.5 \mathrm{mg}$ daily - the lower end of the target daily dose in Parkinson's disease (Constantinescu 2008). However, in clinical practice, it is advised to titrate up to this dose and indeed on initial testing, we found that acute administration of $1.5 \mathrm{mg}$ without prior titration was poorly tolerated, necessitating our decision to use the lower dose of $0.5 \mathrm{mg}$. There are two further limitations; the first is that 16 out of the 18 patients were taking medication for OCD at the time of the study, though the dopaminergic drug treatment effect was present in OCD irrespective of SSRI treatment (high vs low dose). A second possible limitation is the relatively modest sample sizes. The study may have been underpowered to determine clear differences for some group comparisons. A retrospective power analysis however provided reasonable confidence levels for correctly rejecting the null hypothesis $(1-\beta>0.7)$.

In some circumstances, pramipexole impairs behavioral learning, possibly by disrupting phasic dopaminergic signaling, in reinforcement learning tasks (Nagy et al. 2012; Pizzagalli et al. 2008), and other learning or decision making tasks (Haley Gallant et al. 2016). In stimulant-dependent individuals, pramipexole has also been shown to reduce behavioral perseveration during learning tasks (Ersche et al. 2011), a behavior frequently found in OCD (Giele et al. 2016; Hauser et al. 2017; Morris et al. 2017). However, Ersche et al. (2011) found no evidence for normalization of behavioral perseveration in the same set of OCD patients as used in this study. We also observed no effects of dopaminergic drugs on our behavioral choice measures of learning in OCD patients or healthy controls although pramipexole did lengthen response times on reward trials, possibly consistent with previous findings reporting a slowing of reward learning after a single dose of pramipexole (Pizzagalli et al. 2008). In contrast, we did demonstrate that the fMRI reward prediction error signal in the anterior cingulate cortex was sensitive to dopaminergic manipulation in $\mathrm{OCD}$, consistent with previous observations in a similar reinforcement learning task that fMRI measures of learning may be more sensitive than behavioral indices (Murray et al. 2010).

Although there have been no randomized controlled clinical effectiveness trials of amisulpride in OCD, meta-analysis confirms that dopamine receptor antagonists reduce obsessive-compulsive symptoms in SSRI-refractory OCD (Dold et al. 2015; Veale et al. 2014). Whilst the addition of a dopamine receptor antagonist medication is a commonly used augmentation strategy for patients who do not recover completely on first-line treatments (Hirschtritt et al. 2017), the mechanisms through which this leads to clinical improvement are not fully understood. Our data suggest one possible mechanism - remediation of otherwise excessive anterior cingulate error signaling in OCD that is related to the selfregulatory impairments we found in these patients. In contrast, we found no effect of dopaminergic drug treatment on the enhanced positive reward prediction error signals expressed in the nucleus accumbens of these OCD patients, which was not shown however to be related to symptoms in this patient sample.

There have been no clinical trials of pramipexole for OCD, although three small clinical trials have shown evidence that the related drug, dexamphetamine, can be effective in OCD (Insel et al. 1983; Joffe et al. 1991; Koran et al. 2009). Our results may prompt consideration of further research into lowdose $\mathrm{D}_{2 / 3}$ agonist treatment for OCD, although, here, considerable caution, rigorous risk assessment, and very careful experimental design are advised given prior reports that pramipexole may cause or worsen compulsive behaviors in some people (Kolla et al. 2010). Our data are consistent with the potential therapeutic actions of partial $\mathrm{D}_{2 / 3}$ agonists; one such drug, aripiprazole, has already been shown to be effective in augmentation in OCD, with some evidence of efficacy at very low doses (Ercan et al. 2015; Janardhan Reddy et al. 2017; Muscatello et al. 2011; Sayyah et al. 2012; Veale et al. 2014). Additional mechanistic and pragmatic research is warranted to determine which OCD patients are most likely to benefit from dopaminergic treatment.

In summary, these data extend previous evidence of abnormal processing of errors in the fronto-striatal networks in OCD, by showing that the heightened sensitivity of anterior 
cingulate cortex in OCD can be reduced by dopaminergic modulation. This study therefore is the first to provide direct evidence of dopaminergic dysfunction during reward prediction error signaling in OCD.

Funding information The study was supported by an award from GSK to University of Cambridge (RG45422, Principal Investigator TWR), a Wellcome Trust Senior Investigator Award (104631/Z/14/Z) to TWR, by a MRC Clinician Scientist award (G0701911) to GKM, and by a Betty Behrens Research Fellowship of Clare Hall to KDE. The research was conducted in the University of Cambridge Behavioural and Clinical Neuroscience Institute which was supported by a joint award from the Medical Research Council (G1000183) and Wellcome Trust (093875/Z/10Z).

Compliance with ethical standards All volunteers provided written informed consent. The study was approved by the Cambridge Research Ethics Committee.

Conflict of interest Dr. Bullmore is employed half-time by GSK and half-time by University of Cambridge. He holds stock in GSK. Dr. Craig was employed by the University of Cambridge at the time the study was initiated; he is now employed by, and holds stock in, Covance, the drug development business of Laboratory Corporation of America ${ }^{\circledR}$ Holdings (LabCorp). Dr. Fineberg reports the following commercial interests, all outside the research work; in the past 2 years, she has received personal fees for giving lectures from Abbott, Otsuka-Lundbeck, RANZCP, BAP, and Wiley; payment for editorial duties from Taylor and Francis; royalties from Oxford University Press; payment for consultancy from the MHRA; research or educational grants from Shire, ECNP, NIHR, MRC, and Wellcome Trust; and non-financial support from Shire, ECNP, BAP, CINP, International Forum of Mood and Anxiety Disorders, International College of Obsessive Compulsive Spectrum Disorders, and RCPsych. Dr. Robbins discloses consultancy with Cambridge Cognition, Lundbeck, Mundipharma, and Unilever; he receives royalties for CANTAB from Cambridge Cognition and editorial honoraria from Springer Verlag and Elsevier. Dr. Sahakian consults for Cambridge Cognition, Peak (Brainbow), and Mundipharma. Ms. Shabbir is employed by GSK. Drs. Abbott, Ersche, Knolle, Murray, and Suckling declare no competing interests.

Open Access This article is distributed under the terms of the Creative Commons Attribution 4.0 International License (http:// creativecommons.org/licenses/by/4.0/), which permits unrestricted use, distribution, and reproduction in any medium, provided you give appropriate credit to the original author(s) and the source, provide a link to the Creative Commons license, and indicate if changes were made.

\section{References}

Abler B, Walter H, Erk S, Kammerer H, Spitzer M (2006) Prediction error as a linear function of reward probability is coded in human nucleus accumbens. Neuroimage 31:790-795

Abler B, Erk S, Walter H (2007) Human reward system activation is modulated by a single dose of olanzapine in healthy subjects in an event-related, double-blind, placebo-controlled fMRI study. Psychopharmacology 191:823-833

Bernacer J, Corlett PR, Ramachandra P, McFarlane B, Turner DC, Clark L, Robbins TW, Fletcher PC, Murray GK (2013) Methamphetamine-induced disruption of Frontostriatal reward learning signals: relation to psychotic symptoms. Am J Psychiatry 170:1326-1334

BOND A, LADER M (1974) The use of analogue scales in rating subjective feelings. Br J Med Psychol 47:211-218

Brown J, Miller W, Lawendowski L (1999) The self-regulation questionnaire. Innov Clin Pract A Source B 281-289

Constantinescu R (2008) Update on the use of pramipexole in the treatment of Parkinson's disease. Neuropsychiatr Dis Treat 4:337-352

Coukell AJ, Spencer CM, Benfield P (1996) Amisulpride. CNS Drugs 6: $237-256$

Denys D, van der Wee N, Janssen J, De Geus F, Westenberg HG (2004) Low level of dopaminergic D2 receptor binding in obsessivecompulsive disorder. Biol Psychiatry 55:1041-1045

Denys D, Mantione M, Figee M, van den Munckhof P, Koerselman F, Westenberg $\mathrm{H}$ et al (2010) Deep brain stimulation of the nucleus accumbens for treatment-refractory obsessive-compulsive disorder. Arch Gen Psychiatry 67:1061-1068

Dold M, Aigner M, Lanzenberger R, Kasper S (2015) Antipsychotic augmentation of serotonin reuptake inhibitors in treatment-resistant obsessive-compulsive disorder: an update meta-analysis of doubleblind, randomized, placebo-controlled trials. Int J Neuropsychopharmacol 18:pyv047

Eisenegger C, Naef M, Linssen A, Clark L, Gandamaneni PK, Müller U, Robbins TW (2014) Role of dopamine D2 receptors in human reinforcement learning. Neuropsychopharmacology 39:2366-2375

Ercan ES, Ardic UA, Ercan E, Yuce D, Durak S (2015) A promising preliminary study of aripiprazole for treatment-resistant childhood obsessive-compulsive disorder. J Child Adolesc Psychopharmacol 25:580-584

Ermakova AO, Knolle F, Justicia A, Bullmore ET, Jones PB, Robbins TW et al (2018) Abnormal reward prediction-error signalling in antipsychotic naive individuals with first-episode psychosis or clinical risk for psychosis. Neuropsychopharmacology 1

Ersche KD, Roiser JP, Abbott S, Craig KJ, Müller U, Suckling J, Ooi C, Shabbir SS, Clark L, Sahakian BJ, Fineberg NA, Merlo-Pich EV, Robbins TW, Bullmore ET (2011) Response perseveration in stimulant dependence is associated with striatal dysfunction and can be ameliorated by a D 2/3 receptor agonist. Biol Psychiatry 70:754762

Figee M, Vink M, de Geus F, Vulink N, Veltman DJ, Westenberg H et al (2011) Dysfunctional reward circuitry in obsessive-compulsive disorder. Biol Psychiatry 69:867-874

Ford CP (2014) The role of D2-autoreceptors in regulating dopamine neuron activity and transmission. Neuroscience 282:13-22

Frank MJ, Seeberger LC, Reilly RC (2004) By carrot or by stick: cognitive reinforcement learning in parkinsonism. Science (80- ) 306: 1940 LP-1941943

Giele CL, van den Hout MA, Engelhard IM, Dek ECP, Toffolo MBJ, Cath DC (2016) Perseveration induces dissociative uncertainty in obsessive-compulsive disorder. J Behav Ther Exp Psychiatry 52:110

Gillan CM, Robbins TW (2014) Goal-directed learning and obsessivecompulsive disorder. Philos Trans R Soc B 369:20130475

Gillan CM, Fineberg NA, Robbins TW (2017) A trans-diagnostic perspective on obsessive-compulsive disorder. Psychol Med 1-21. https://doi.org/10.1017/S0033291716002786

Goodman WK, Price LH, Rasmussen SA, Mazure C, Fleischmann RL, Hill CL, Heninger GR, Charney DS (1989) The Yale-Brown obsessive compulsive scale: I. Development, use, and reliability. Arch Gen Psychiatry 46:1006-1011

Grace AA, Bunney BS (1983) Intracellular and extracellular electrophysiology of nigral dopaminergic neurons-1. Identification and characterization. Neuroscience 10:301-315

Haley Gallant AV, Seergobin KN, MacDonald PA (2016) Pramipexole impairs stimulus-response learning in healthy young adults. Front Neurosci 10 
Hammer A, Kordon A, Heldmann M, Zurowski B, Münte TF (2009) Brain potentials of conflict and error-likelihood following errorful and errorless learning in obsessive-compulsive disorder. PLoS One 4:e6553

Hauser TU, Iannaccone R, Dolan RJ, Ball J, Hättenschwiler J, Drechsler R, Rufer M, Brandeis D, Walitza S, Brem S (2017) Increased frontostriatal reward prediction errors moderate decision making in obsessive-compulsive disorder. Psychol Med 47:1246-1258

Hesse S, Müller U, Lincke T, Barthel H, Villmann T, Angermeyer MC, Sabri O, Stengler-Wenzke K (2005) Serotonin and dopamine transporter imaging in patients with obsessive-compulsive disorder. Psychiatry Res Neuroimaging 140:63-72

Hester R, Nandam LS, O'Connell RG, Wagner J, Strudwick M, Nathan PJ et al (2012) Neurochemical enhancement of conscious error awareness. J Neurosci 32:2619-2627. https://doi.org/10.1523/ jneurosci.4052-11.2012

Hirschtritt ME, Bloch MH, Mathews CA (2017) Obsessive-compulsive disorder: advances in diagnosis and treatment. JAMA 317:13581367

Horst NK, Jupp B, Roberts AC, Robbins TW (2019) D2 receptors and cognitive flexibility in marmosets: tri-phasic dose-response effects of intra-striatal quinpirole on serial reversal performance. Neuropsychopharmacology 44:564-571

Howell DC (2009) Statistical methods for psychology. Ann Phys (N Y) 6th:324-325

Insel TR, Hamilton JA, Guttmacher LB, Murphy DL (1983) DAmphetamine in obsessive-compulsive disorder. Psychopharmacology 80:231-235

Janardhan Reddy YC, Sundar AS, Narayanaswamy JC, Math SB (2017) Clinical practice guidelines for obsessive-compulsive disorder. Indian J Psychiatry 59:S74-S90

Jenkinson M, Beckmann CF, Behrens TEJ, Woolrich MW, Smith SM (2012) FSL. Neuroimage 62:782-790

Jocham G, Klein TA, Ullsperger M (2011) Dopamine-mediated reinforcement learning signals in the striatum and ventromedial prefrontal cortex underlie value-based choices. J Neurosci 31:1606-1613

Joffe RT, Swinson RP, Levitt AJ (1991) Acute psychostimulant challenge in primary obsessive-compulsive disorder. J Clin Psychopharmacol 11:237-241

Kehagia AA, Murray GK, Robbins TW (2010) Learning and cognitive flexibility: frontostriatal function and monoaminergic modulation. Curr Opin Neurobiol 20:169-192

Kolla BP, Mansukhani MP, Barraza R, Bostwick JM (2010) Impact of dopamine agonists on compulsive behaviors: a case series of pramipexole-induced pathological gambling. Psychosomatics 51: 271-273

Koran LM, Aboujaoude E, Gamel NN (2009) Double-blind study of dextroamphetamine versus caffeine augmentation for treatmentresistant obsessive-compulsive disorder. J Clin Psychiatry 70: $1530-1535$

Mathews CA, Perez VB, Delucchi KL, Mathalon DH (2012) Errorrelated negativity in individuals with obsessive-compulsive symptoms: toward an understanding of hoarding behaviors. Biol Psychol 89:487-494

Morein-Zamir S, Papmeyer M, Gillan CM, Crockett MJ, Fineberg NA, Sahakian BJ, Robbins TW (2013) Punishment promotes response control deficits in obsessive-compulsive disorder: evidence from a motivational go/no-go task. Psychol Med 43:391-400

Moresco RM, Pietra L, Henin M, Panzacchi A, Locatelli M, Bonaldi L, Carpinelli A, Gobbo C, Bellodi L, Perani D, Fazio F (2007) Fluvoxamine treatment and D2 receptors: a pet study on OCD drug-naive patients. Neuropsychopharmacology 32:197-205

Morris LS, Baek K, Voon V (2017) Distinct cortico-striatal connections with subthalamic nucleus underlie facets of compulsivity. Cortex 88: $143-150$
Murray G, Corlett P, Clark L, Pessiglione M, Blackwell A, Honey G et al (2008) Substantia nigra / ventral tegmental reward prediction error disruption in psychosis. Mol Psychiatry 13:1-18

Murray GK, Corlett PR, Fletcher PC (2010) The neural underpinnings of associative learning in health and psychosis: how can performance be preserved when brain responses are abnormal? Schizophr Bull 36:465-471

Muscatello MRA, Bruno A, Pandolfo G, Micò U, Scimeca G, Romeo VM, Santoro V, Settineri S, Spina E, Zoccali RA (2011) Effect of aripiprazole augmentation of serotonin reuptake inhibitors or clomipramine in treatment-resistant obsessive-compulsive disorder. J Clin Psychopharmacol 31:174-179

Nagy H, Levy-Gigi E, Somlai Z, Takáts A, Bereczki D, Kéri S (2012) The effect of dopamine agonists on adaptive and aberrant salience in Parkinson's disease. Neuropsychopharmacology 37:950-958

Nielen MM, Den Boer JA, Smid H (2009) Patients with obsessivecompulsive disorder are impaired in associative learning based on external feedback. Psychol Med 39:1519-1526

O'doherty J, Dayan P, Schultz J, Deichmann R, Friston K, Dolan RJ (2004) Dissociable roles of ventral and dorsal striatum in instrumental conditioning. Science (80- ) 304:452-454

Olver JS, O'Keefe G, Jones GR, Burrows GD, Tochon-Danguy HJ, Ackermann U et al (2009) Dopamine D 1 receptor binding in the striatum of patients with obsessive-compulsive disorder. J Affect Disord 114:321-326

Olver JS, O'Keefe G, Jones GR, Burrows GD, Tochon-Danguy HJ, Ackermann U et al (2010) Dopamine D 1 receptor binding in the anterior cingulate cortex of patients with obsessive-compulsive disorder. Psychiatry Res Neuroimaging 183:85-88

Pagnoni G, Zink CF, Montague PR, Berns GS (2002) Activity in human ventral striatum locked to errors of reward prediction. Nat Neurosci 5:97-98

Pauls AM, O’Daly OG, Rubia K, Riedel WJ, Williams SCR, Mehta MA (2012) Methylphenidate effects on prefrontal functioning during attentional-capture and response inhibition. Biol Psychiatry 72: 142-149. https://doi.org/10.1016/j.biopsych.2012.03.028

Perani D, Garibotto V, Gorini A, Moresco RM, Henin M, Panzacchi A, Matarrese M, Carpinelli A, Bellodi L, Fazio F (2008) In vivo PET study of 5HT 2A serotonin and D 2 dopamine dysfunction in drugnaive obsessive-compulsive disorder. Neuroimage 42:306-314

Perrault GH, Depoortere R, Morel E, Sanger DJ, Scatton B (1997) Psychopharmacological profile of amisulpride: an antipsychotic drug with presynaptic D2/D3 dopamine receptor antagonist activity and limbic selectivity. J Pharmacol Exp Ther 280:73-82

Pessiglione M, Seymour B, Flandin G, Dolan RJ, Frith CD (2006) Dopamine-dependent prediction errors underpin reward-seeking behaviour in humans. Nature 442:1042-1045

Pizzagalli DA, Evins AE, Schetter EC, Frank MJ, Pajtas PE, Santesso DL, Culhane M (2008) Single dose of a dopamine agonist impairs reinforcement learning in humans: behavioral evidence from a laboratory-based measure of reward responsiveness. Psychopharmacology 196:221-232

Rodriguez PF, Aron AR, Poldrack RA (2006) Ventral-striatal/nucleusaccumbens sensitivity to prediction errors during classification learning. Hum Brain Mapp 27:306-313

Rosenzweig P, Canal M, Patat A, Bergougnan L, Zieleniuk I, Bianchetti G (2002) A review of the pharmacokinetics, tolerability and pharmacodynamics of amisulpride in healthy volunteers. Hum Psychopharmacol 17:1-13

Sayyah M, Sayyah M, Boostani H, Ghaffari SM, Hoseini A (2012) Effects of aripiprazole augmentation in treatment-resistant obsessive-compulsive disorder (a double blind clinical trial). Depress Anxiety 29:850-854

Schultz W, Dickinson A (2000) Neuronal coding of prediction errors. Annu Rev Neurosci 23:473-500 
Schultz W, Dayan P, Montague PR (1997) A neural substrate of prediction and reward. Science (80- ) 275:1593-1599

Sesia T, Bizup B, Grace AA (2013) Evaluation of animal models of obsessive-compulsive disorder: correlation with phasic dopamine neuron activity. Int J Neuropsychopharmacol 16:1295-1307

Stern ER, Welsh RC, Fitzgerald KD, Gehring WJ, Lister JJ, Himle JA, Abelson JL, Taylor SF (2011) Hyperactive error responses and altered connectivity in ventromedial and frontoinsular cortices in obsessive-compulsive disorder. Biol Psychiatry 69:583-591

Ursu S, Stenger VA, Shear MK, Jones MR, Carter CS (2003) Overactive action monitoring in obsessive-compulsive disorder: evidence from functional magnetic resonance imaging. Psychol Sci 14:347-353

Van Der Wee NJ, Stevens H, Hardeman JA, Mandl RC, Denys DA, Van Megen HJ et al (2004) Enhanced dopamine transporter density in psychotropic-naive patients with obsessive-compulsive disorder shown by [123I] $\beta$-CIT SPECT. Am J Psychiatry 161:2201-2206
Veale D, Miles S, Smallcombe N, Ghezai H, Goldacre B, Hodsoll J (2014) Atypical antipsychotic augmentation in SSRI treatment refractory obsessive-compulsive disorder: a systematic review and meta-analysis. BMC Psychiatry 14:317

Wright CE, Sisson TL, Ichhpurani AK, Peters GR (1997) Steady-state pharmacokinetic properties of pramipexole in healthy volunteers. J Clin Pharmacol 37:520-525

Publisher's note Springer Nature remains neutral with regard to jurisdictional claims in published maps and institutional affiliations. 